\title{
Quasi associated continued fractions and Hankel determinants of Dixon elliptic functions via Sumudu transform
}

\author{
Adem Kilicman $^{\mathrm{a}, *}$, Rathinavel Silambarasan ${ }^{\mathrm{b}}$, Omer Altun $^{\mathrm{c}}$ \\ ${ }^{a}$ Department of Mathematics, Faculty of Science, Universiti Putra Malaysia, 43400 UPM Serdang, Selangor, Malaysia. \\ ${ }^{b}$ M. Tech IT-Networking, Department of Information Technology, School of Information Technology and Engineering, VIT University, \\ Vellore, Tamilnadu, India. \\ ${ }^{c}$ Department of Mathematics and Institute for Mathematical research, Universiti Putra Malaysia, 43400 UPM Serdang, Selangor, \\ Malaysia.
}

Communicated by X.-J. Yang

\begin{abstract}
In this work, Sumudu transform of Dixon elliptic functions for higher arbitrary powers $\operatorname{sm}^{N}(x, \alpha) ; N \geqslant 1, \operatorname{sm}^{N}(x, \alpha) c^{2}(x, \alpha) ;$ $N \geqslant 0$ and $\operatorname{sm}^{N}(x, \alpha) \mathrm{cm}^{2}(x, \alpha) ; N \geqslant 0$ by considering modulus $\alpha \neq 0$ is obtained as three term recurrences and hence expanded as product of quasi associated continued fractions where the coefficients are functions of $\alpha$. Secondly the coefficients of quasi associated continued fractions are used for Hankel determinants calculations by connecting the formal power series (Maclaurin series) and associated continued fractions. (C)2017 All rights reserved.
\end{abstract}

Keywords: Dixon elliptic functions, quasi associated continued fractions, Hankel determinants, Sumudu transform, three term recurrence.

2010 MSC: 11A55, 11C20, 33E05, 44A10.

\section{Introduction}

Jacobi elliptic functions $s n(x, k), c n(x, k)$ and $d n(x, k)$, there ratios were applied with Laplace transform to calculate Hankel determinants from their continued fractions in [26] and Fourier series of those functions were used to derive the different ways of writing square and triangular numbers [26]. Continued fractions related to Hahn and orthogonal polynomials from the results of Laplace transform of Jacobi elliptic functions such as difference equations derived in [16]. Bimodular Jacobi elliptic functions treated with Laplace transform to solve as continued fractions and by using modular transformation results were traced back to Jacobi elliptic functions in [9]. Determinants of Bernoulli numbers, coefficients of Maclaurin series of Jacobi elliptic functions were calculated through continued fractions in [2] and orthogonal polynomials from Fourier series of Jacobi elliptic functions in [8].

On studying the cubic curve $x^{3}+y^{3}-3 \alpha x y=1$ in [11] gave raise to elliptic functions $s m(x, \alpha)$ and $\operatorname{cm}(x, \alpha)$ named after Dixon whose functions are doubly periodic, related properties and examples given

\footnotetext{
*Corresponding author

Email addresses: akilic@upm.edu.my (Adem Kilicman), silambu_vel@yahoo.co.in (Rathinavel Silambarasan), omeraltun11@yahoo.com (Omer Altun)
}

doi:10.22436/jnsa.010.07.49 
in [12]. Similar cubic curve on $\alpha=0$ is studied and led to elliptic functions in [13]. $\operatorname{sm}(x, 0)$ and $\mathrm{cm}(x, 0)$ are applied to conformal mapping study and addition, multiplication formulae pertaining those maps given in [1]. Dixon elliptic functions are used for representing factorial of numbers given in [3] and related to Fermat cubes, combinatorics given in [10]. Laplace transform is applied to Dixon functions and extended to various types of continued fractions in [9]. In [22] Dixon functions connecting trefoil curve and Weierstrass elliptic functions $[22,23]$ have been described.

Sumudu transform successor of Laplace transform in problem solving possess unique properties namely unit, scale and dimension preserving where other transforms does not satisfy, studied to solve fractional Maxwell's equations in [21]. Sumudu transform is applied for various engineering problems for their solutions in $[4,14,15,18]$ and solved with perturbation, decomposition methods in $[25,28]$. New definition of Sumudu transform for trigonometric functions and infinite series expansions is derived in [5]. Symbolic C++ and Maple procedure for Sumudu transform is given in [6]. Sumudu transform of Dumont bimodular Jacobi elliptic functions from which Sumudu transform of $\tan (a x), \sec (a x)$ and their products for arbitrary powers are derived as product of associated continued fractions and their Hankel determinants in [7]. Sumudu transform [4-7, 14, 15, 18-21, 25, 28, 29] of $f(x)$ is defined in the set

$$
A=\left\{f(x)\left|\exists M, \tau_{1}, \tau_{2}>0,\right| f(x) \mid<M e^{\frac{|x|}{\tau_{j}}}, \text { if } x \in(-1)^{j} \times[0, \infty)\right\},
$$

given by integral equation

$$
\mathrm{S}[\mathrm{f}(\mathrm{x})](\mathrm{u})=\int_{0}^{\infty} e^{-x} f(u x) d x=1 / u \int_{0}^{\infty} e^{-x / u} f(x) d x ; u \in\left(-\tau_{1}, \tau_{2}\right) .
$$

In [32] Novel Integral Transform (NIT) is defined and shown as dual for Laplace, Laplace Carson and Sumudu transforms. $\mathrm{N}_{\mathrm{I}}[f(x)](\gamma)=1 / \gamma^{2} \mathbb{S}[f(x)](u)_{\mathfrak{u}=1 / \gamma}$. Here $\gamma$ is the NIT variable, and extended to $(-\infty, \infty)$ and showed dual to Fourier transform in [33].

In this paper Sumudu transform is applied to Dixon Elliptic Functions (DEF) namely $\operatorname{sm}^{N}(x, \alpha)$, $\mathrm{sm}^{\mathrm{N}}(x, \alpha) \operatorname{cm}(x, \alpha)$ and $\mathrm{sm}^{\mathrm{N}}(x, \alpha) \mathrm{cm}^{2}(x, \alpha)$ to show their results as product of Quasi Associated Continued Fractions (QACF). Coefficients of obtained QACF are employed for Hankel determinants derivations using Heliermann correspondence connecting formal power series to associated continued fractions.

\section{Preliminaries}

Considering the cubic curve $x^{3}+y^{3}-3 \alpha x y=1 ;(\alpha \neq-1)$ in [11] derived the two set of functions $\operatorname{sm}(x, \alpha)$ and $\operatorname{cm}(x, \alpha)$ which are doubly periodic also showed they are elliptic. Derivative of DEF ([11, Eqs. (1) and (3), page 171] and [9, Eqs. (1.18) and (1.19), page 9])

$$
\frac{d}{d x} \operatorname{sm}(x, \alpha)=\operatorname{cm}^{2}(x, \alpha)-\alpha \operatorname{sm}(x, \alpha) \text { and } \frac{d}{d x} \operatorname{cm}(x, \alpha)=-\operatorname{sm}^{2}(x, \alpha)+\alpha c m(x, \alpha),
$$

with ([9, Eq. (1.21), page 10]),

$$
\operatorname{sm}(0, \alpha)=1 \text { and } \operatorname{cm}(0, \alpha)=1,
$$

and the functions satisfy the cubic curve mentioned above named Pythagorean theorem ([11, Eq. (2), page 171] and [9, Eq. (1.22), page 10])

$$
\operatorname{sm}^{3}(x, \alpha)+\mathrm{cm}^{3}(x, \alpha)-3 \alpha \operatorname{sm}(x, \alpha) c \mathfrak{c}(x, \alpha)=1 .
$$

Continued fraction notation taken from ([17, Eq. (2.1.4 b), page 18] and ([24, Eq. (1.2.5'), page 8])

$$
\mathbf{K}_{n=1}^{\infty} \frac{a_{n}}{b_{n}}=\frac{a_{1}}{b_{1}+} \frac{a_{2}}{b_{2}+} \frac{a_{3}}{b_{3}+\cdots}=\frac{a_{1}}{b_{1}+\frac{a_{2}}{b_{2}+\frac{a_{3}}{b_{3}+\frac{a_{4}}{b_{4}+\ddots}}} .}
$$


Definition 2.1. Let $a=\left\{a_{n}\right\}, b=\left\{b_{n}\right\}$ and $u$ is an indeterminate, then the continued fraction of following form is called associated continued fraction ([17, Eq. (7.2.1), page 241]), [30] and ([31, Eq. (54.1), page 208]):

$$
1+\frac{a_{0} u}{1+b_{0} u+} \mathbf{K}_{n=1}^{\infty} \frac{-a_{n} u}{1+b_{n} u}
$$

Remark 2.2. The continued fraction of the form

$$
\frac{a_{0} u}{c_{0}+b_{0} u+} \mathbf{K}_{n=1}^{\infty} \frac{-a_{n} u}{c_{n}+b_{n} u}
$$

is called QACF. Moreover the coefficients $a_{0}, b_{0}, c_{0}, a_{n}, b_{n}$ and $c_{n} ; n \geqslant 1$ are functions of $u$ rather constants.

Definition 2.3. Let $c=\left\{c_{v}\right\}_{v=1}^{\infty}$ be a sequence in $\mathbb{C}$. Then the following $m \times m$ matrices are defined $[9,17,26]$, whose determinants are denoted by respective $H_{m}^{(n)}$ and $\chi_{m}$ :

$$
\begin{gathered}
H_{m}^{(n)}=H_{m}^{(n)}\left(c_{v}\right)=\operatorname{det}\left(\begin{array}{ccccc}
c_{n} & c_{n+1} & \cdots & c_{m+n-2} & c_{n+m-1} \\
c_{n+1} & c_{n+2} & \cdots & c_{m+n-1} & c_{m+n} \\
\vdots & \vdots & \ddots & \vdots & \vdots \\
c_{m+n-1} & c_{m+n} & \cdots & c_{2 m+n-3} & c_{2 m+n-2}
\end{array}\right), \\
\chi_{m}=\chi_{m}\left(c_{v}\right)=\operatorname{det}\left(\begin{array}{ccccc}
c_{1} & c_{2} & \cdots & c_{m-1} & c_{m+1} \\
c_{2} & c_{3} & \cdots & c_{m} & c_{m+2} \\
\vdots & \vdots & \ddots & \vdots & \vdots \\
c_{m} & c_{m+1} & \cdots & c_{2 m-2} & c_{2 m}
\end{array}\right)
\end{gathered}
$$

Remark 2.4. The matrix for $\chi_{m}$ is obtained from the matrix for $\mathrm{H}_{m+1}^{(1)}$ by deleting the last row and next to last column $[9,17,26]$. For $n=1, H_{1}^{(1)}=c_{1}$ and $\chi_{1}=c_{2}$. Determinants $H_{m}^{(n)}$ and $\chi_{m}$ [27] are named as persymmetric determinants (or) Turanian determinants (or) Hankel determinants.

The relation between formal power series and its associated continued fractions is given as lemma [9], ([17, Theorem 7.14, pp 244-248]), [26].

Lemma 2.5. When an associated continued fraction converges to formal power series,

$$
1+\sum_{v=1}^{\infty} c_{v} z^{v}=1+\frac{a_{0} z}{1+b_{0} z+} \mathbf{K}_{n=1}^{\infty} \frac{-a_{n} z^{2}}{1+b_{n} z^{\prime}}, \quad\left(a_{n} \neq 0\right)
$$

then,

$$
\mathrm{H}_{m}^{(1)}\left(\left[c_{v}\right]\right) \neq 0, \quad a_{m}=\frac{H_{m}^{(1)} H_{m-2}^{(1)}}{\left(H_{m-1}^{(1)}\right)^{2}} \quad \text { and } \quad b_{m}=\frac{\chi_{m-1}}{H_{m-1}^{(1)}}-\frac{\chi_{m}}{H_{m}^{(1)}}, \quad(m \geqslant 1),
$$

where $\mathrm{H}_{-1}^{(1)}=\mathrm{H}_{0}^{(1)}=1$ and $\chi_{0}=0$. Conversely if (2.5) is true then (2.4) is true. Also,

$$
H_{m}^{(1)}\left(\left[c_{v}\right]\right)=\prod_{j=1}^{m} a_{j}^{m+1-j}=\prod_{j=0}^{m-1} a_{j}^{m-j}, \quad \text { and } \quad \frac{\chi_{m}\left(\left[c_{v}\right]\right)}{H_{m}^{(1)}\left(\left[c_{v}\right]\right)}=-\sum_{j=1}^{m} b_{j}, \quad(m \geqslant 1) .
$$

\section{Main results 1: Sumudu transform of Dixon elliptic functions $(\alpha \neq 0)$}

In [9] Laplace transform is applied for DEF and obtained QACF for up to third power of $\operatorname{sm}(x, \alpha)$ and second power of $\operatorname{sm}(x, \alpha) \mathrm{cm}(x, \alpha)$ results given. In this paper, we generalize the previous work to 
arbitrary powers and show its back traceable to results of [9]. Followed by, Hankel determinants are derived for the QACF using Lemma 2.5 by assuming $c_{0}=c_{n} ; n \geqslant 1=1$. Applying Sumudu transform (1.1) to higher powers of DEF, main results of this paper are given in following three theorems.

Theorem 3.1. Sumudu transform of DEF $\operatorname{sm}^{N}(x, \alpha) ; N \geqslant 1$ is given by QACF:

(i)

(ii)

$$
\mathrm{S}[\operatorname{sm}(x, \alpha)](u)=\frac{(1-5 \alpha u) u}{F_{1}^{*}+4 u^{3}+} \frac{-6 E_{4} u^{6}}{F_{4}+G_{4} u^{3}+} \mathbf{K}_{n=2}^{\infty} \frac{-E_{3 n+1} H_{3 n-2} u^{6}}{F_{3 n+1}+G_{3 n+1} u^{3}} .
$$

$$
\mathrm{S}\left[\operatorname{sm}^{2}(x, \alpha)\right](u)=\frac{2(1-7 \alpha u) u^{2}}{F_{2}+G_{2} u^{3}+} \mathbf{K}_{n=1}^{\infty} \frac{-E_{3 n+2} H_{3 n-1} u^{6}}{F_{3 n+2}+G_{3 n+2} u^{3}} .
$$

(iii) Let $\mathrm{N}=3,6,9,12, \cdots$

$$
S\left[\operatorname{sm}^{N}(x, \alpha)\right](u)=\prod_{i=1}^{\frac{N}{3}} \frac{E_{3 i} u^{3}}{F_{3 i}+G_{3 i} u^{3}+} \prod_{n=1}^{\infty} \frac{-E_{3 n+3 i} H_{3 n+3 i-3} u^{6}}{F_{3 n+3 i}+G_{3 n+3 i} u^{3}} .
$$

(iv) Let $\mathrm{N}=4,7,10,13, \cdots$

$$
\begin{aligned}
\mathrm{S}\left[\operatorname{sm}^{\mathrm{N}}(x, \alpha)\right](u)= & \frac{(1-5 \alpha u) u}{F_{1}^{*}+4 u^{3}+} \frac{-6 E_{4} u^{6}}{F_{4}+G_{4} u^{3}+} \mathbf{K}_{n=2}^{\infty} \frac{-E_{3 n+1} H_{3 n-2} u^{6}}{F_{3 n+1}+G_{3 n+1} u^{3}} \\
& \times \prod_{i=1}^{\frac{N-1}{3}} \frac{E_{3 i+1} u^{3}}{F_{3 i+1}+G_{3 i+1} u^{3}+} \prod_{n=1}^{\infty} \frac{-E_{3 n+3 i+1} H_{3 n+3 i-2} u^{6}}{F_{3 n+3 i+1}+G_{3 n+3 i+1} u^{3}} .
\end{aligned}
$$

(v) Let $\mathrm{N}=5,8,11,14, \cdots$

$$
\begin{aligned}
\mathrm{S}\left[\operatorname{sm}^{\mathrm{N}}(x, \alpha)\right](u)= & \frac{2(1-7 \alpha u)}{\mathrm{F}_{2}+\mathrm{G}_{2} \mathrm{u}^{3}+} \mathbf{K}_{n=1}^{\infty} \frac{-\mathrm{E}_{3 n+2} \mathrm{H}_{3 n-1} \mathrm{u}^{6}}{\mathrm{~F}_{3 n+2}+\mathrm{G}_{3 n+2} \mathrm{u}^{3}} \\
& \times \prod_{i=1}^{\frac{N-2}{3}} \frac{\mathrm{E}_{3 i+2} \mathrm{u}^{3}}{\mathrm{~F}_{3 i+2}+\mathrm{G}_{3 i+2} \mathrm{u}^{3}+} \mathbf{K}_{n=1}^{\infty} \frac{-\mathrm{E}_{3 n+3 i+2} \mathrm{H}_{3 n+3 i-1} \mathrm{u}^{6}}{\mathrm{~F}_{3 n+3 i+2}+\mathrm{G}_{3 n+3 i+2} \mathrm{u}^{3}}
\end{aligned}
$$

where $E_{(.)}, F_{(.)}, G_{(.)}$and $H_{(.)}$are polynomials in $u$ and $\alpha$ defined in equations (3.10), (3.11), (3.12), (3.13) respectively and $\mathrm{F}_{1}^{*}=\mathrm{F}_{1}^{*}(\mathrm{u}, \alpha)=(1-5 \alpha u)(1-2 \alpha u)(1+\alpha u)[9]$.

Proof. For $\mathrm{N} \geqslant 0$ :

$$
\begin{gathered}
\mathrm{S}\left[\operatorname{sm}^{\mathrm{N}}(x, \alpha)\right](u)=A_{N}=\int_{0}^{\infty} e^{-x} \operatorname{sm}^{\mathrm{N}}(x u, \alpha) d x, \\
\mathrm{~S}\left[\operatorname{sm}^{\mathrm{N}}(x, \alpha) \operatorname{cm}(x, \alpha)\right](u)=\mathrm{B}_{\mathrm{N}}=\int_{0}^{\infty} e^{-x} \operatorname{sm}^{\mathrm{N}}(x u, \alpha) c m(x u, \alpha) d x, \\
\mathrm{~S}\left[\operatorname{sm}^{\mathrm{N}}(x, \alpha) \mathrm{cm}^{2}(x, \alpha)\right](u)=\mathrm{C}_{\mathrm{N}}=\int_{0}^{\infty} e^{-x} \operatorname{sm}^{\mathrm{N}}(x u, \alpha) \mathrm{cm}^{2}(x u, \alpha) \mathrm{d} x .
\end{gathered}
$$

Integrating by parts, (3.6) reduces to the following difference equations using (2.1), (2.2), (2.3) in $A_{N}$ and $\mathrm{C}_{\mathrm{N}-1}$ with $A_{0}=1$ :

$$
\begin{aligned}
A_{1} & =u C_{0}-\alpha u A_{1}, \\
A_{2} & =2 u C_{1}-2 \alpha u A_{2}, \\
A_{3} & =3 u C_{2}-3 \alpha u A_{3}, \\
& \vdots \\
A_{N} & =N u C_{N-1}-N \alpha u A_{N} .
\end{aligned}
$$

Solving for $A_{N}$ using recurrences of (3.7) and (3.8) leads to QACF: 


$$
\frac{A_{N}}{A_{N-3}}=\frac{E_{N} u^{3}}{F_{N}+G_{N} u^{3}-H_{N} u^{3} \frac{A_{N+3}}{A_{N}}}, \quad(N \geqslant 3),
$$

where,

$$
\begin{aligned}
E_{N} & =E_{N}(u, \alpha)=(N-2)(N-1) N(1-(2 N+3) \alpha u), \quad(N \geqslant 3), \\
F_{N}=F_{N}(u, \alpha) & =(1-(2 N-3) \alpha u)(1-(2 N+3) \alpha u)(1+N \alpha u)(1-2 N \alpha u), \quad(N \geqslant 2), \\
G_{N}=G_{N}(u, \alpha) & =(1-(2 N+3) \alpha u)(N-1)^{2} N+(1-(2 N-3) \alpha u) N(N+1)^{2}, \quad(N \geqslant 2), \\
H_{N}=H_{N}(u, \alpha) & =N(N+1)(N+2)(1-(2 N-3) \alpha u), \quad(N \geqslant 3) .
\end{aligned}
$$

For $\mathrm{N}=1,2$ and 3

$$
\begin{aligned}
& A_{1}=\frac{(1-5 \alpha u) u}{(1-5 \alpha u)(1-2 \alpha u)(1+\alpha u)+4 u^{3}-6 u^{3} \frac{A_{4}}{A_{1}}} \\
& A_{2}=\frac{2(1-7 \alpha u) u^{2}}{F_{2}+G_{2} u^{3}-H_{2} u^{3} \frac{A_{5}}{A_{2}}} \\
& A_{3}=\frac{E_{3} u^{3}}{F_{3}+G_{3} u^{3}-H_{3} u^{3} \frac{A_{6}}{A_{3}}} .
\end{aligned}
$$

Now (3.1) and (3.2) are immediate consequences from respective (3.14) and (3.15) iterating with (3.9). Equations (3.3), (3.4), (3.5) are obtained by continuous iteration of (3.9) starting with (3.16) and after mathematical calculations and simplification completes the proof.

Remark 3.2. Multiplying $u$ to (3.1) and (3.2) gives the result in [9] ([9, Theorems 22 and 23, page 65]). Multiplying (3.3) with $u$ and for $N=3$ gives the result in [9] ([9, Theorem 24, page 65]). Results derived in equations (3.3), (3.4), (3.5) are new with the existing literature.

Theorem 3.3. Sumudu transform of DEF $\operatorname{sm}^{\mathrm{N}}(x, \alpha) \mathrm{cm}(x, \alpha) ; \mathrm{N} \geqslant 0$ is given by QACF:

(i)

$$
\mathrm{S}[\mathrm{cm}(x, \alpha)]=\frac{\mathrm{P}_{0}^{*}}{\mathrm{Q}_{0}^{*}+2 \mathrm{u}^{3}+} \frac{-6 \mathrm{P}_{3} \mathrm{u}^{6}}{\mathrm{Q}_{3}+\mathrm{R}_{3} \mathrm{u}^{3}+} \mathbf{K}_{n=2}^{\infty} \frac{-S_{3 n-3} \mathrm{P}_{3 n} \mathrm{u}^{6}}{\mathrm{Q}_{3 n}+\mathrm{R}_{3 n} \mathrm{u}^{3}}
$$

(ii)

$$
\mathrm{S}[\operatorname{sm}(x, \alpha) \operatorname{cm}(x, \alpha)]=\frac{\mathrm{P}_{1}^{*} u}{\mathrm{Q}_{1}^{*}+12 \mathrm{u}^{3}+} \mathbf{K}_{n=1}^{\infty} \frac{-S_{3 n-2} P_{3 n+1} u^{6}}{Q_{3 n+1}+R_{3 n+1} u^{3}}
$$

(iii)

$$
\mathrm{S}\left[\operatorname{sm}^{2}(x, \alpha) \operatorname{cm}(x, \alpha)\right]=\frac{2 P_{2}^{*} u^{2}}{Q_{2}+R_{2} u^{3}+} \mathbf{K}_{n=1}^{\infty} \frac{-S_{3 n-1} P_{3 n+2} u^{6}}{Q_{3 n+2}+R_{3 n+2} u^{3}}
$$

(iv) Let $\mathrm{N}=3,6,9,12, \cdots$

$$
\begin{aligned}
\mathrm{S}\left[\operatorname{sm}^{\mathrm{N}}(x, \alpha) \operatorname{cm}(x, \alpha)\right]= & \frac{\mathrm{P}_{0}^{*}}{\mathrm{Q}_{0}^{*}+2 u^{3}+} \frac{-6 P_{3} u^{6}}{\mathrm{Q}_{3}+R_{3} u^{3}+} \mathbf{K}_{n=2}^{\infty} \frac{-S_{3 n-3} P_{3 n} u^{6}}{Q_{3 n}+R_{3 n} u^{3}} \\
& \times \prod_{i=1}^{\frac{N}{3}} \frac{P_{3 i} u^{3}}{Q_{3 i}+R_{3 i} u^{3}+} K_{n=1}^{\infty} \frac{-S_{3 n+3 i-3} P_{3 n+3 i} u^{6}}{Q_{3 n+3 i}+R_{3 n+3 i} u^{3}}
\end{aligned}
$$


(v) Let $\mathrm{N}=4,7,10,13, \cdots$

$$
\begin{aligned}
\mathrm{S}\left[\operatorname{sm}^{\mathrm{N}}(x, \alpha) \operatorname{cm}(x, \alpha)\right]= & \frac{\mathrm{P}_{1}^{*} u}{\mathrm{Q}_{1}^{*}+12 u^{3}+} \mathbf{K}_{n=1}^{\infty} \frac{-S_{3 n-2} P_{3 n+1} u^{6}}{Q_{3 n+1}+R_{3 n+1} u^{3}} \\
& \times \prod_{i=1}^{\frac{N-1}{3}} \frac{P_{3 i+1} u^{3}}{Q_{3 i+1}+R_{3 i+1} u^{3}+} K_{n=1}^{\infty} \frac{-S_{3 n+3 i-2} P_{3 n+3 i+1} u^{6}}{Q_{3 n+3 i+1}+R_{3 n+3 i+1} u^{3}} .
\end{aligned}
$$

(vi) Let $\mathrm{N}=5,8,11,14, \cdots$

$$
\begin{aligned}
\mathrm{S}\left[\operatorname{sm}^{\mathrm{N}}(x, \alpha) \operatorname{cm}(x, \alpha)\right]= & \frac{2 \mathrm{P}_{2}^{*} \mathrm{u}^{2}}{\mathrm{Q}_{2}+\mathrm{R}_{2} \mathrm{u}^{3}+} \mathbf{K}_{n=1}^{\infty} \frac{-S_{3 n-1} P_{3 n+2} \mathrm{u}^{6}}{\mathrm{Q}_{3 n+2}+R_{3 n+2} \mathrm{u}^{3}} \\
& \times \prod_{i=1}^{\frac{N-2}{3}} \frac{\mathrm{P}_{3 i+2} \mathrm{u}^{3}}{\mathrm{Q}_{3 i+2}+\mathrm{R}_{3 i+2} \mathrm{u}^{3}+} \mathbf{K}_{n=1}^{\infty} \frac{-S_{3 n+3 i-1} P_{3 n+3 i+2} u^{6}}{Q_{3 n+3 i+2}+R_{3 n+3 i+2} u^{3}},
\end{aligned}
$$

where $P_{(.)}, Q_{(.)}, R_{(.)}$and $S_{(.)}$are polynomials in $u$ and $\alpha$ given by equations (3.24), (3.25), (3.26), (3.27), respectively [9] and $\mathrm{P}_{0}^{*}, \mathrm{Q}_{0}^{*}, \mathrm{P}_{1}^{*}, \mathrm{Q}_{1}^{*}$ and $\mathrm{P}_{2}^{*}$ are polynomials in $\mathrm{u}$ and $\alpha$ given by equations (3.31), (3.32), (3.33), (3.34), (3.35), respectively.

Proof. Using equations (2.1), (2.2), (2.3), evaluating by parts, (3.7) reduces to the following difference equations in $A_{N-1}$ and $B_{N}$ :

$$
\begin{aligned}
\mathrm{B}_{0} & =1-u A_{2}+\alpha u B_{0} \\
B_{1} & =u-2 u A_{3}+3 \alpha u B_{1} \\
B_{2} & =2 u A_{1}-3 u A_{4}+5 \alpha u B_{2} \\
B_{3} & =3 u A_{2}-4 u A_{5}+7 \alpha u B_{3} \\
& \vdots \\
B_{N} & =N u A_{N-1}-(N+1) u A_{N+2}+(2 N+1) \alpha u B_{N}
\end{aligned}
$$

Solving for $B_{N}$ using recurrences of (3.6) and (3.8) leads to QACF:

$$
\frac{B_{N}}{B_{N-3}}=\frac{P_{N} u^{3}}{Q_{N}+R_{N} u^{3}-S_{N} u^{3} \frac{B_{N+3}}{B_{N}}}, \quad(N \geqslant 3),
$$

where,

$$
\begin{aligned}
& \mathrm{P}_{\mathrm{N}}=\mathrm{P}_{\mathrm{N}}(\mathrm{u}, \alpha)=(\mathrm{N}-2)(\mathrm{N}-1) \mathrm{N}(1-(2 \mathrm{~N}+4) \alpha u)(1+(\mathrm{N}+2) \alpha u), \quad(\mathrm{N} \geqslant 3), \\
& \mathrm{Q}_{\mathrm{N}}=\mathrm{Q}_{\mathrm{N}}(\mathrm{u}, \alpha)=(1-(2 \mathrm{~N}-2) \alpha u)(1+(\mathrm{N}-1) \alpha u)(1-(2 \mathrm{~N}+4) \alpha u) \\
& \times(1+(N+2) \alpha u)(1-(2 N+1) \alpha u), \quad(N \geqslant 2), \\
& \mathrm{R}_{\mathrm{N}}=\mathrm{R}_{\mathrm{N}}(\mathrm{u}, \alpha)=(\mathrm{N}-1) \mathrm{N}^{2}(1-(2 \mathrm{~N}+4) \alpha \mathrm{u})(1+(\mathrm{N}+2) \alpha \mathrm{u}) \\
& +(N+1)^{2}(N+2)(1-(2 N-2) \alpha u)(1+(N-1) \alpha u), \quad(N \geqslant 2), \\
& S_{N}=S_{N}(u, \alpha)=(N+1)(N+2)(N+3)(1-(2 N-2) \alpha u)(1+(N-1) \alpha u), \quad(N \geqslant 1) \text {. }
\end{aligned}
$$

For $\mathrm{N}=0,1$ and 2 :

$$
\begin{aligned}
& \mathrm{B}_{0}=\frac{\mathrm{P}_{0}^{*}}{\mathrm{Q}_{0}^{*}+2 \mathrm{u}^{3}-6 \mathrm{u}^{3} \frac{\mathrm{B}_{3}}{\mathrm{~B}_{0}}}, \\
& \mathrm{~B}_{1}=\frac{\mathrm{P}_{1}^{*} \mathrm{u}}{\mathrm{Q}_{1}^{*}+12 \mathrm{u}^{3}-\mathrm{S}_{1} \mathrm{u}^{3} \frac{\mathrm{B}_{4}}{\mathrm{~B}_{1}}},
\end{aligned}
$$




$$
\mathrm{B}_{2}=\frac{2 \mathrm{P}_{2}^{*} \mathrm{u}^{2}}{\mathrm{Q}_{2}+\mathrm{R}_{2} \mathrm{u}^{3}-\mathrm{S}_{2} \mathrm{u}^{3} \frac{\mathrm{B}_{5}}{\mathrm{~B}_{2}}}
$$

where,

$$
\begin{aligned}
P_{0}^{*}=P_{0}^{*}(u, \alpha) & =(1-4 \alpha u)(1+2 \alpha u), \\
Q_{0}^{*}=Q_{0}^{*}(u, \alpha) & =(1-4 \alpha u)(1+2 \alpha u)(1-\alpha u), \\
P_{1}^{*}=P_{1}^{*}(u, \alpha) & =(1-6 \alpha u)(1+3 \alpha u), \\
Q_{1}^{*}=Q_{1}^{*}(u, \alpha) & =(1-6 \alpha u)(1+3 \alpha u)(1-3 \alpha u), \\
P_{2}^{*}=P_{2}^{*}(u, \alpha) & =(1-8 \alpha u)(1+4 \alpha u) .
\end{aligned}
$$

Hence equations (3.17), (3.18), (3.19) follow from (3.28), (3.29), (3.30) respectively iterating with (3.23). And equations (3.20), (3.21), (3.22) are obtained from continuous iteration with (3.23) and simplification which completes the proof.

Remark 3.4. Multiplying u to equations (3.17), (3.18), (3.19) gives results in [9] ([9, Theorems 25-27, pp 66-67]). Equations (3.20), (3.21), (3.22) are new with existing literature.

Theorem 3.5. Sumudu transform of DEF $\operatorname{sm}^{\mathrm{N}}(x, \alpha) \mathrm{cm}^{2}(x, \alpha) ; \mathrm{N} \geqslant 0$ is given by QACF:

(i)

$$
\mathrm{S}\left[\mathrm{cm}^{2}(x, \alpha)\right]=\frac{\mathrm{T}_{0}^{*}}{\mathrm{~V}_{0}^{*}+\mathrm{W}_{0}^{*} \mathrm{u}^{3}+} \frac{-\mathrm{X}_{0}^{*} \mathrm{~T}_{3} \mathrm{u}^{6}}{\mathrm{~V}_{3}+\mathrm{W}_{3} \mathrm{u}^{3}+} \mathbf{K}_{n=2}^{\infty} \frac{-X_{3 n-3} T_{3 n} \mathrm{u}^{6}}{\mathrm{~V}_{3 n}+W_{3 n} u^{3}}
$$

(ii)

$$
\mathrm{S}\left[\operatorname{sm}(x, \alpha) \mathrm{cm}^{2}(x, \alpha)\right]=\frac{\mathrm{T}_{1}^{*}}{V_{1}^{*}+W_{1}^{*} u^{3}+} \frac{-X_{1}^{*} T_{4} u^{6}}{V_{4}+W_{4} u^{3}+} \mathbf{K}_{n=2}^{\infty} \frac{-X_{3 n-2} T_{3 n+1} u^{6}}{V_{3 n+1}+W_{3 n+1} u^{3}}
$$

(iii)

$$
\mathrm{S}\left[\operatorname{sm}^{2}(x, \alpha) \mathrm{cm}^{2}(x, \alpha)\right]=\frac{\mathrm{T}_{2}^{*} \mathrm{u}^{2}}{\mathrm{~V}_{2}+\mathrm{W}_{2} \mathrm{u}^{3}+} \mathbf{K}_{n=1}^{\infty} \frac{-X_{3 n-1} T_{3 n+2} u^{6}}{V_{3 n+2}+W_{3 n+2} u^{3}} .
$$

(iv) Let $\mathrm{N}=3,6,9,12, \cdots$

$$
\begin{aligned}
\mathrm{S}\left[\mathrm{sm}^{\mathrm{N}}(x, \alpha) \mathrm{cm}^{2}(x, \alpha)\right]= & \frac{\mathrm{T}_{0}^{*}}{\mathrm{~V}_{0}^{*}+W_{0}^{*} u^{3}+} \frac{-X_{0}^{*} T_{3} u^{6}}{V_{3}+W_{3} u^{3}+} \mathbf{K}_{n=2}^{\infty} \frac{-X_{3 n-3} T_{3 n} u^{6}}{V_{3 n}+W_{3 n} u^{3}} \\
& \times \prod_{i=1}^{\frac{N}{3}} \frac{T_{3 i} u^{3}}{V_{3 i}+W_{3 i} u^{3}+} \mathbf{K}_{n=1}^{\infty} \frac{-X_{3 n+3 i-3} T_{3 n+3 i} u^{6}}{V_{3 n+3 i}+W_{3 n+3 i} u^{3}} .
\end{aligned}
$$

(v) Let $\mathrm{N}=4,7,10,13, \cdots$

$$
\begin{aligned}
\mathrm{S}\left[\operatorname{sm}^{\mathrm{N}}(x, \alpha) \mathrm{cm}^{2}(x, \alpha)\right]= & \frac{\mathrm{T}_{1}^{*}}{\mathrm{~V}_{1}^{*}+W_{1}^{*} u^{3}+} \frac{-X_{1}^{*} T_{4} u^{6}}{V_{4}+W_{4} u^{3}+} \mathbf{K}_{n=2}^{\infty} \frac{-X_{3 n-2} T_{3 n+1} u^{6}}{V_{3 n+1}+W_{3 n+1} u^{3}} \\
& \times \prod_{i=1}^{\frac{N-1}{3}} \frac{T_{3 i+1} u^{3}}{V_{3 i+1}+W_{3 i+1} u^{3}+} \mathbf{K}_{n=1}^{\infty} \frac{-X_{3 n+3 i-2} T_{3 n+3 i+1} u^{6}}{V_{3 n+3 i+1}+W_{3 n+3 i+1} u^{3}}
\end{aligned}
$$


(vi) Let $\mathrm{N}=5,8,11,14, \cdots$

$$
\begin{aligned}
\mathrm{S}\left[\operatorname{sm}^{\mathrm{N}}(x, \alpha) \mathrm{cm}^{2}(x, \alpha)\right]= & \frac{T_{2}^{*} u^{2}}{V_{2}+W_{2} u^{3}+} \mathbf{K}_{n=1}^{\infty} \frac{-X_{3 n-1} T_{3 n+2} u^{6}}{V_{3 n+2}+W_{3 n+2} u^{3}} \\
& \times \prod_{i=1}^{\frac{N-2}{3}} \frac{T_{3 i+2} u^{3}}{V_{3 i+2}+W_{3 i+2} u^{3}+} \mathbf{K}_{n=1}^{\infty} \frac{-X_{3 n+3 i-1} T_{3 n+3 i+2} u^{6}}{V_{3 n+3 i+2}+W_{3 n+3 i+2} u^{3}}
\end{aligned}
$$

where $T_{(.)}, V_{(.)}, W_{(.)}$and $X_{(.)}$are polynomials in $u$ and $\alpha$ given by equations (3.43), (3.44), (3.45), (3.46), respectively and $\mathrm{T}_{0}^{*}, \mathrm{~V}_{0}^{*}, \mathrm{~W}_{0}^{*}, \mathrm{X}_{0}^{*}, \mathrm{~T}_{1}^{*}, \mathrm{~V}_{1}^{*}, \mathrm{~W}_{1}^{*}, \mathrm{~W}_{1}^{*}$ and $\mathrm{T}_{2}^{*}$ are polynomials in $\mathrm{u}$ and $\alpha$ given by equations (3.50), (3.51), (3.52), (3.53), (3.54), (3.55), (3.56), (3.57), (3.58), respectively.

Proof. Integrating by parts and using (2.1), (2.2), (2.3), equation (3.8) reduces to the following difference equations in $\mathrm{B}_{\mathrm{N}-1}$ and $\mathrm{C}_{\mathrm{N}}$ :

$$
\begin{aligned}
C_{0} & =1-2 u B_{2}+2 \alpha u C_{0} \\
C_{1} & =u B_{0}-3 u B_{3}+4 \alpha u C_{1} \\
C_{2} & =2 u B_{1}-4 u B_{4}+6 \alpha u C_{2} \\
C_{3} & =3 u B_{2}-5 u B_{5}+8 \alpha u C_{3} \\
& \vdots \\
C_{N} & =N u B_{N-1}-(N+2) u B_{N+2}+(2 N+2) \alpha u C_{N}
\end{aligned}
$$

Solving for $C_{N}$ using recurrences of (3.6) and (3.7) yields QACF only in $C_{N}$ :

$$
\frac{C_{N}}{C_{N-3}}=\frac{T_{N} u^{3}}{V_{N}+W_{N} u^{3}-X_{N} u^{3} \frac{C_{N+3}}{C_{N}}}, \quad(N \geqslant 3),
$$

where,

$$
\begin{aligned}
\mathrm{T}_{\mathrm{N}}=\mathrm{T}_{\mathrm{N}}(\mathrm{u}, \alpha)= & (\mathrm{N}-2)(\mathrm{N}-1) \mathrm{N}(1+(\mathrm{N}+1) \alpha u)(1+(\mathrm{N}+4) \alpha u) \\
& \times(1-(2 \mathrm{~N}+5) \alpha u), \quad(\mathrm{N} \geqslant 3), \\
\mathrm{V}_{\mathrm{N}}=\mathrm{V}_{\mathrm{N}}(\mathrm{u}, \alpha)= & (1+(\mathrm{N}+1) \alpha u)(1+(\mathrm{N}-2) \alpha u)(1-(2 \mathrm{~N}-1) \alpha u) \\
& \times(1-(2 \mathrm{~N}+2) \alpha u)(1+(\mathrm{N}+4) \alpha u)(1-(2 \mathrm{~N}+5) \alpha u), \quad(\mathrm{N} \geqslant 1), \\
\mathrm{W}_{\mathrm{N}}=\mathrm{W}_{\mathrm{N}}(\mathrm{u}, \alpha)= & (1+(\mathrm{N}-2) \alpha u)(1-(2 \mathrm{~N}+5) \alpha u)(1+(\mathrm{N}+4) \alpha u) \mathrm{N}^{2}(\mathrm{~N}+1) \\
& +(1+(\mathrm{N}-2) \alpha u)(1-(2 \mathrm{~N}-1) \alpha u) \\
& \times(1+(\mathrm{N}+4) \alpha u)(\mathrm{N}+1)(\mathrm{N}+2)^{2}, \quad(\mathrm{~N} \geqslant 1), \\
\mathrm{X}_{\mathrm{N}}=\mathrm{X}_{\mathrm{N}}(\mathrm{u}, \alpha)= & (\mathrm{N}+2)(\mathrm{N}+3)(\mathrm{N}+4)(1+(\mathrm{N}+1) \alpha u) \\
& \times(1+(\mathrm{N}-2) \alpha u)(1-(2 \mathrm{~N}-1) \alpha u), \quad(\mathrm{N} \geqslant 1) .
\end{aligned}
$$

For $\mathrm{N}=0,1$ and 2 :

$$
\begin{aligned}
& \mathrm{C}_{0}=\frac{\mathrm{T}_{0}^{*}}{\mathrm{~V}_{0}^{*}+\mathrm{W}_{0}^{*} \mathrm{u}^{3}-\mathrm{X}_{0}^{*} \mathrm{u}^{3} \frac{\mathrm{C}_{3}}{\mathrm{C}_{0}}}, \\
& \mathrm{C}_{1}=\frac{\mathrm{T}_{1}^{*} \mathrm{u}}{\mathrm{V}_{1}^{*}+\mathrm{W}_{1}^{*} \mathrm{u}^{3}-\mathrm{X}_{1}^{*} \mathrm{u}^{3} \frac{\mathrm{C}_{4}}{\mathrm{C}_{1}}}, \\
& \mathrm{C}_{2}=\frac{\mathrm{T}_{2}^{*} \mathrm{u}^{2}}{\mathrm{~V}_{2}+\mathrm{W}_{2} \mathrm{u}^{3}-\mathrm{X}_{2} \mathrm{u}^{3} \frac{\mathrm{C}_{5}}{\mathrm{C}_{2}}},
\end{aligned}
$$


where,

$$
\begin{aligned}
\mathrm{T}_{0}^{*}=\mathrm{T}_{0}^{*}(u, \alpha) & =(1+\alpha u)(1+4 \alpha u)(1-5 \alpha u), \\
\mathrm{V}_{0}^{*}=\mathrm{V}_{0}^{*}(u, \alpha) & =(1+\alpha u)(1+4 \alpha u)(1-5 \alpha u)(1-2 \alpha u), \\
\mathrm{W}_{0}^{*}=\mathrm{W}_{0}^{*}(\mathrm{u}, \alpha) & =4(1+4 \alpha u), \\
\mathrm{X}_{0}^{*}=X_{0}^{*}(u, \alpha) & =24(1+\alpha u), \\
\mathrm{T}_{1}^{*}=\mathrm{T}_{1}^{*}(u, \alpha) & =(1+2 \alpha u)(1-7 \alpha u)(1+5 \alpha u), \\
\mathrm{V}_{1}^{*}=\mathrm{V}_{1}^{*}(u, \alpha) & =(1+2 \alpha u)(1-7 \alpha u)(1+5 \alpha u)(1-\alpha u)(1-4 \alpha u), \\
\mathrm{W}_{1}^{*}=\mathrm{W}_{1}^{*}(\mathrm{u}, \alpha) & =2(1-7 \alpha u)(1+5 \alpha u)+18(1-\alpha u)(1+5 \alpha u), \\
X_{1}^{*}=X_{1}^{*}(u, \alpha) & =60(1-\alpha u)(1+2 \alpha u), \\
\mathrm{T}_{2}^{*}=\mathrm{T}_{2}^{*}(u, \alpha) & =2(1+3 \alpha u)(1-9 \alpha u)(1+6 \alpha u) .
\end{aligned}
$$

Now equations (3.36), (3.37), (3.38) follow from respective equations (3.47), (3.48), (3.49) iterating with (3.42). Equations (3.39), (3.40), (3.41) are obtained by iterating continuously (3.42) and simplifying completes the proof.

Remark 3.6. All the results in Theorem 3.5, equations (3.36), (3.37), (3.38), (3.39), (3.40), (3.41) are new with the existing literature.

Remark 3.7. NIT of DEF $\operatorname{sm}^{\mathrm{N}}(x, \alpha) ; \mathrm{N} \geqslant 1, \operatorname{sm}^{\mathrm{N}}(x, \alpha) \operatorname{cm}(x, \alpha) ; \mathrm{N} \geqslant 0$ and $\operatorname{sm}^{\mathrm{N}}(x, \alpha) \mathrm{cm}^{2}(x, \alpha) ; \mathrm{N} \geqslant 0$ can be obtained from Theorems 3.1, 3.3 and 3.5 respectively by replacing $u=1 / \gamma$ and multiplying with $1 / \gamma^{2}$ and simplifying yields the NIT of DEF.

\section{Main results 2: Hankel determinants derivations}

In [9] QACF is given for Laplace transform of DEF $(\alpha \neq 0)$ and for Hankel determinants calculations to those QACF ([9, first query, pp 73-73]) are given as future directions. In this section Hankel determinants pertaining from Section 3 using Lemma 2.5 are calculated. Main results of this section are given in the following three theorems.

Theorem 4.1. Hankel determinants corresponding to Theorem 3.1 are given by,

(i)

$$
\begin{aligned}
& \mathrm{H}_{1}^{(1)}\left([\operatorname{sm}(x, \alpha)]_{3 v+1}\right)=(1-5 \alpha u), \\
& \mathrm{H}_{2}^{(1)}\left([\operatorname{sm}(x, \alpha)]_{3 v+1}\right)=6(1-5 \alpha u)^{2} E_{4}, \\
& H_{m}^{(1)}\left([\operatorname{sm}(x, \alpha)]_{3 v+1}\right)=6^{(m-1)}(1-5 \alpha u)^{m} \prod_{j=0}^{m-1} E_{3 j+4}^{(m-j-1)} \prod_{j=1}^{m-2} H_{3 j+1}^{(m-j-1)}, \quad m \geqslant 3, \\
& \frac{\chi_{1}\left([\operatorname{sm}(x, \alpha)]_{3 v+1}\right)}{H_{1}^{(1)}\left([\operatorname{sm}(x, \alpha)]_{3 v+1}\right)}=-4, \\
& \frac{\chi_{m}\left([\operatorname{sm}(x, \alpha)]_{3 v+1}\right)}{H_{m}^{(1)}\left([\operatorname{sm}(x, \alpha)]_{3 v+1}\right)}=-\left[4+\sum_{j=1}^{m-1} G_{3 j+1}\right], \quad m \geqslant 2 .
\end{aligned}
$$

(ii)

$$
\begin{aligned}
& H_{1}^{(1)}\left(\left[\operatorname{sm}^{2}(x, \alpha)\right]_{3 v+2}\right)=2(1-7 \alpha u), \\
& H_{m}^{(1)}\left(\left[\operatorname{sm}^{2}(x, \alpha)\right]_{3 v+2}\right)=2^{m}(1-7 \alpha u)^{m} \prod_{j=1}^{m-1} E_{3 j+2}^{(m-j)} H_{3 j-1}^{(m-j)}, \quad m \geqslant 2,
\end{aligned}
$$




$$
\frac{\chi_{m}\left(\left[\mathrm{sm}^{2}(x, \alpha)\right]_{3 v+2}\right)}{\mathrm{H}_{m}^{(1)}\left(\left[\mathrm{sm}^{2}(x, \alpha)\right]_{3 v+2}\right)}=-\sum_{j=1}^{m} G_{3 j-1}, \quad m \geqslant 1 .
$$

(iii) Let $\mathrm{N}=3,6,9,12, \cdots$

$$
\begin{aligned}
& H_{1}^{(1)}\left(\left[\operatorname{sm}^{N}(x, \alpha)\right]_{3 v+N}\right)=E_{3 i} \\
& H_{m}^{(1)}\left(\left[\operatorname{sm}^{N}(x, \alpha)\right]_{3 v+N}\right)=E_{3 i}^{m} \prod_{j=1}^{m-1} E_{3 j+3 i}^{(m-j)} H_{3 j+3 i-3}^{(m-j)}, \quad m \geqslant 2, \\
& \frac{\chi_{m}\left(\left[s m^{N}(x, \alpha)\right]_{3 v+N}\right)}{H_{m}^{(1)}\left(\left[\operatorname{sm}^{N}(x, \alpha)\right]_{3 v+N}\right)}=-\sum_{j=1}^{m} G_{3 j+3 i-3}, \quad m \geqslant 1,
\end{aligned}
$$

where $i=1,2,3, \cdots, \frac{N}{3}$.

(iv) Let $\mathrm{N}=4,7,10,13, \cdots$

$$
\begin{aligned}
& H_{1}^{(1)}\left(\left[\mathrm{sm}^{\mathrm{N}}(x, \alpha)\right]_{3 v+N}\right)=E_{3 i+1} \\
& H_{m}^{(1)}\left(\left[\mathrm{sm}^{\mathrm{N}}(x, \alpha)\right]_{3 v+N}\right)=E_{3 i+1}^{m} \prod_{j=1}^{m-1} E_{3 j+3 i+1}^{(m-j)} H_{3 j+3 i-2}^{(m-j)}, \quad m \geqslant 2, \\
& \frac{\chi_{m}\left(\left[\mathrm{sm}^{\mathrm{N}}(x, \alpha)\right]_{3 v+N}\right)}{H_{m}^{(1)}\left(\left[\mathrm{sm}^{N}(x, \alpha)\right]_{3 v+N}\right)}=-\sum_{j=1}^{m} G_{3 j+3 i-2,}, \quad m \geqslant 1,
\end{aligned}
$$

where $i=1,2,3, \cdots, \frac{N-1}{3}$.

(v) Let $\mathrm{N}=5,8,11,14, \cdots$

$$
\begin{aligned}
& H_{1}^{(1)}\left(\left[\mathrm{sm}^{\mathrm{N}}(x, \alpha)\right]_{3 v+N}\right)=E_{3 i+2} \\
& H_{m}^{(1)}\left(\left[\mathrm{sm}^{\mathrm{N}}(x, \alpha)\right]_{3 v+N}\right)=E_{3 i+2}^{m} \prod_{j=1}^{m-1} E_{3 j+3 i+2}^{(m-j)} H_{3 j+3 i-1}^{(m-j)}, \quad m \geqslant 2, \\
& \frac{\chi_{m}\left(\left[\mathrm{sm}^{\mathrm{N}}(x, \alpha)\right]_{3 v+N}\right)}{H_{m}^{(1)}\left(\left[s m^{N}(x, \alpha)\right]_{3 v+N}\right)}=-\sum_{j=1}^{m} G_{3 j+3 i-1}, \quad m \geqslant 1,
\end{aligned}
$$

where $i=1,2,3, \cdots, \frac{\mathrm{N}-2}{3}$.

Here $E_{(.)}, G_{(.)}$and $H_{(.)}$are given by (3.10), (3.12) and (3.13), respectively.

Proof. Maclaurin series of $s m^{\mathrm{N}}(x, \alpha) ; \mathrm{N} \geqslant 1$ is given by,

$$
\operatorname{sm}^{\mathrm{N}}(x, \alpha)=\sum_{v=0}^{\infty} \frac{\left[\mathrm{sm}^{\mathrm{N}}(x, \alpha)\right]_{3 v+N} \chi^{3 v+N}}{(3 v+N) !}, \quad \mathrm{N} \geqslant 1,
$$

where $\left[\operatorname{sm}^{\mathrm{N}}(x, \alpha)\right]_{3 v+\mathrm{N}}$ are Maclaurin series coefficients in polynomials of $\alpha$. Next assume

$$
(1-5 \alpha u)(1-2 \alpha u)(1+\alpha u) \equiv 1,
$$

in (3.1), (3.4) and $\mathrm{F}_{\mathrm{N}} ;(\mathrm{N} \geqslant 2) \equiv 1$ in Theorem 3.1, so that it satisfies Lemma 2.5. Now applying coefficients of Theorem 3.1 in (2.6) of Lemma 2.5 gives the Hankel determinants for equations (3.1), (3.2), (3.3), (3.4), (3.5) of Theorem 3.1 by respective enumerates in Theorem 4.1. 
Remark 4.2. Substituting $\alpha=0$ in equations (4.1), (4.2), (4.3), (4.4), (4.5), (4.6), (4.7), (4.8), (4.9), (4.10), (4.11) for $\mathrm{N}=3$ in Theorem 4.1 gives the Hankel determinants in [9] ([9, Theorems 10-12, pp 48-50]), respectively.

Theorem 4.3. Hankel determinants corresponding to Theorem 3.3 are given by,

(i)

$$
\begin{aligned}
& \mathrm{H}_{1}^{(1)}\left([\mathrm{cm}(x, \alpha)]_{3 v}\right)=P_{0}^{*}, \\
& H_{2}^{(1)}\left([\mathrm{cm}(x, \alpha)]_{3 v}\right)=6\left(P_{0}^{*}\right)^{2} P_{3}, \\
& H_{m}^{(1)}\left([\mathrm{cm}(x, \alpha)]_{3 v}\right)=6^{(m-1)}\left(P_{0}^{*}\right)^{m} P_{3}^{(m-1)} \prod_{j=1}^{m-2} S_{3 j}^{(m-j-1)} P_{3 j+3}^{(m-j-1)}, \quad m \geqslant 3, \\
& \frac{\chi_{1}\left([\mathrm{~cm}(x, \alpha)]_{3 v}\right)}{H_{1}^{(1)}\left([\mathrm{cm}(x, \alpha)]_{3 v}\right)}=-2, \\
& \frac{\chi_{m}\left([\mathrm{~cm}(x, \alpha)]_{3 v}\right)}{H_{m}^{(1)}\left([\mathrm{cm}(x, \alpha)]_{3 v}\right)}=-\left[2+\sum_{j=1}^{m-1} R_{3 j}\right], \quad m \geqslant 2 .
\end{aligned}
$$

(ii)

$$
\begin{aligned}
& H_{1}^{(1)}\left([\operatorname{sm}(x, \alpha) \mathrm{cm}(x, \alpha)]_{3 v+1}\right)=P_{1}^{*}, \\
& H_{m}^{(1)}\left([\operatorname{sm}(x, \alpha) \operatorname{cm}(x, \alpha)]_{3 v+1}\right)=\left(P_{1}^{*}\right)^{m} \prod_{j=1}^{m-1} S_{3 j-2}^{(m-j)} P_{3 j+1}^{(m-j)}, \quad m \geqslant 2, \\
& \frac{\chi_{1}\left([\operatorname{sm}(x, \alpha) \operatorname{cm}(x, \alpha)]_{3 v+1}\right)}{H_{1}^{(1)}\left([\operatorname{sm}(x, \alpha) \operatorname{cm}(x, \alpha)]_{3 v+1}\right)}=-12, \\
& \frac{\chi_{m}\left([\operatorname{sm}(x, \alpha) \operatorname{cm}(x, \alpha)]_{3 v+1}\right)}{H_{m}^{(1)}\left([\operatorname{sm}(x, \alpha) \operatorname{cm}(x, \alpha)]_{3 v+1}\right)}=-\left[12+\sum_{j=1}^{m-1} R_{3 j+1}\right], \quad m \geqslant 2 .
\end{aligned}
$$

(iii)

$$
\begin{aligned}
& H_{1}^{(1)}\left(\left[\operatorname{sm}^{2}(x, \alpha) c m(x, \alpha)\right]_{3 v+2}\right)=2 P_{2}^{*}, \\
& H_{m}^{(1)}\left(\left[\operatorname{sm}^{2}(x, \alpha) c m(x, \alpha)\right]_{3 v+2}\right)=2^{m}\left(P_{2}^{*}\right)^{m} \prod_{j=1}^{m-1} S_{3 j-1}^{(m-j)} P_{3 j+2}^{(m-j)}, \quad m \geqslant 2, \\
& \frac{\chi_{m}\left(\left[\operatorname{sm}^{2}(x, \alpha) c m(x, \alpha)\right]_{3 v+2}\right)}{H_{m}^{(1)}\left(\left[\operatorname{sm}^{2}(x, \alpha) c m(x, \alpha)\right]_{3 v+2}\right)}=-\sum_{j=1}^{m} R_{3 j-1}, \quad m \geqslant 1 .
\end{aligned}
$$

(iv) Let $\mathrm{N}=3,6,9,12, \cdots$

$$
\begin{aligned}
& H_{1}^{(1)}\left(\left[\operatorname{sm}^{N}(x, \alpha) c m(x, \alpha)\right]_{3 v+N}\right)=P_{3 i}, \\
& H_{m}^{(1)}\left(\left[\operatorname{sm}^{N}(x, \alpha) c m(x, \alpha)\right]_{3 v+N}\right)=\left(P_{3 i}\right)^{m} \prod_{j=1}^{m-1} S_{3 j+3 i-3}^{(m-j)} P_{3 j+3 i}^{(m-j)}, \quad m \geqslant 2, \\
& \frac{\chi_{m}\left(\left[\operatorname{sm}^{N}(x, \alpha) c m(x, \alpha)\right]_{3 v+N}\right)}{H_{m}^{(1)}\left(\left[\operatorname{sm}^{N}(x, \alpha) c m(x, \alpha)\right]_{3 v+N}\right)}=-\sum_{j=1}^{m} R_{3 j+3 i-3}, \quad m \geqslant 1,
\end{aligned}
$$

where $i=1,2,3, \cdots, \frac{N}{3}$. 
(v) Let $\mathrm{N}=4,7,10,13, \cdots$

$$
\begin{aligned}
& H_{1}^{(1)}\left(\left[\operatorname{sm}^{N}(x, \alpha) c m(x, \alpha)\right]_{3 v+N}\right)=P_{3 i+1}, \\
& H_{m}^{(1)}\left(\left[\operatorname{sm}^{N}(x, \alpha) c m(x, \alpha)\right]_{3 v+N}\right)=\left(P_{3 i+1}\right)^{m} \prod_{j=1}^{m-1} S_{3 j+3 i-2}^{(m-j)} P_{3 j+3 i+1}^{(m-j)} ; m \geqslant 2, \\
& \frac{\chi_{m}\left(\left[s m^{N}(x, \alpha) c m(x, \alpha)\right]_{3 v+N}\right)}{H_{m}^{(1)}\left(\left[\operatorname{sm}^{N}(x, \alpha) c m(x, \alpha)\right]_{3 v+N}\right)}=-\sum_{j=1}^{m} R_{3 j+3 i-2}, \quad m \geqslant 1,
\end{aligned}
$$

where $i=1,2,3, \cdots, \frac{N-1}{3}$.

(vi) Let $\mathrm{N}=5,8,11,14, \cdots$

$$
\begin{aligned}
& H_{1}^{(1)}\left(\left[\operatorname{sm}^{N}(x, \alpha) c m(x, \alpha)\right]_{3 v+N}\right)=P_{3 i+2} \\
& H_{m}^{(1)}\left(\left[\operatorname{sm}^{N}(x, \alpha) c m(x, \alpha)\right]_{3 v+N}\right)=\left(P_{3 i+2}\right)^{m} \prod_{j=1}^{m-1} S_{3 j+3 i-1}^{(m-j)} P_{3 j+3 i+2}^{(m-j)}, \quad m \geqslant 2, \\
& \frac{\chi_{m}\left(\left[\operatorname{sm}^{N}(x, \alpha) c m(x, \alpha)\right]_{3 v+N}\right)}{H_{m}^{(1)}\left(\left[\operatorname{sm}^{N}(x, \alpha) c m(x, \alpha)\right]_{3 v+N}\right)}=-\sum_{j=1}^{m} R_{3 j+3 i-1}, \quad m \geqslant 1
\end{aligned}
$$

where $i=1,2,3, \cdots, \frac{N-2}{3}$.

Here $P_{(.)}, R_{(.)}, S_{(.)}, P_{0}^{*}, P_{1}^{*}$ and $P_{2}^{*}$ are given by respective equations (3.24), (3.26), (3.27), (3.31), (3.33) and (3.35).

Proof. Maclaurin series of $\operatorname{sm}^{\mathrm{N}}(x, \alpha) \mathrm{cm}(x, \alpha) ; \mathrm{N} \geqslant 0$ is given by,

$$
\operatorname{sm}^{\mathrm{N}}(x, \alpha) \operatorname{cm}(x, \alpha)=\sum_{v=0}^{\infty} \frac{\left[\mathrm{sm}^{\mathrm{N}}(x, 0) \mathrm{cm}(x, 0)\right]_{3 v+\mathrm{N}} x^{3 v+N}}{(3 v+\mathrm{N}) !}, \quad \mathrm{N} \geqslant 0,
$$

where $\left[\mathrm{sm}^{\mathrm{N}}(x, \alpha) \mathrm{cm}(x, \alpha)\right]_{3 v+\mathrm{N}} ; \mathrm{N} \geqslant 0$ denotes Maclaurin series coefficients in polynomials of $\alpha$. Now assume $(1-4 \alpha u)(1+2 \alpha u)(1-\alpha u) \equiv 1$ in (3.17) and (3.20), $(1-6 \alpha u)(1+3 \alpha u)(1-3 \alpha u) \equiv 1$ in (3.18) and (3.21), $Q_{N} ; N \geqslant 2 \equiv 1$ in Theorem 3.3 so that it satisfies Lemma 2.5. Applying coefficients of Theorem 3.3 in (2.6) of Lemma 2.5 gives the Hankel determinants for equations (3.17), (3.18), (3.19), (3.20), (3.21), (3.22) by respective enumerates of Theorem 4.3 .

Remark 4.4. Substituting $\alpha=0$ in equations (4.12), (4.13), (4.14), (4.15), (4.16), (4.17), (4.18), (4.19), (4.20), (4.21), (4.22), (4.23), Theorem 4.3 gives the results in [9] ([9, Theorems 13-15, pp 51-53]), respectively.

Theorem 4.5. Hankel determinants corresponding to Theorem 3.5 are given by,

(i)

$$
\begin{aligned}
& \mathrm{H}_{1}^{(1)}\left(\left[\mathrm{cm}^{2}(x, \alpha)\right]_{3 v}\right)=\mathrm{T}_{0}^{*}, \\
& \mathrm{H}_{2}^{(1)}\left(\left[\mathrm{cm}^{2}(x, \alpha)\right]_{3 v}\right)=\left(\mathrm{T}_{0}^{*}\right)^{2} \mathrm{X}_{0}^{*} \mathrm{~T}_{3}, \\
& H_{m}^{(1)}\left(\left[\mathrm{cm}^{2}(x, \alpha)\right]_{3 v}\right)=\left(T_{0}^{*}\right)^{m}\left(X_{0}^{*}\right)^{m-1}\left(T_{3}\right)^{m-1} \prod_{j=1}^{m-2} x_{3 j}^{(m-j-1)} T_{3 j+3}^{(m-j-1)}, \quad m \geqslant 3, \\
& \frac{\chi_{1}\left(\left[\mathrm{~cm}^{2}(x, \alpha)\right]_{3 v}\right)}{H_{1}^{(1)}\left(\left[\mathrm{cm}^{2}(x, \alpha)\right]_{3 v}\right)}=-W_{0}^{*} \\
& \frac{\chi_{m}\left(\left[\mathrm{~cm}^{2}(x, \alpha)\right]_{3 v}\right)}{H_{m}^{(1)}\left(\left[\mathrm{cm}^{2}(x, \alpha)\right]_{3 v}\right)}=-\left[W_{0}^{*}+\sum_{j=1}^{m-1} W_{3 j}\right], \quad m \geqslant 2 .
\end{aligned}
$$


(ii)

$$
\begin{aligned}
& H_{1}^{(1)}\left(\left[\operatorname{sm}(x, \alpha) \mathrm{cm}^{2}(x, \alpha)\right]_{3 v+1}\right)=\mathrm{T}_{1}^{*}, \\
& \mathrm{H}_{2}^{(1)}\left(\left[\operatorname{sm}(x, \alpha) \mathrm{cm}^{2}(x, \alpha)\right]_{3 v+1}\right)=\left(\mathrm{T}_{1}^{*}\right)^{2} X_{1}^{*} \mathrm{~T}_{4}, \\
& \mathrm{H}_{\mathrm{m}}^{(1)}\left(\left[\operatorname{sm}(x, \alpha) \mathrm{cm}^{2}(x, \alpha)\right]_{3 v+1}\right)=\left(\mathrm{T}_{1}^{*}\right)^{\mathrm{m}}\left(X_{1}^{*}\right)^{\mathrm{m}-1}\left(\mathrm{~T}_{4}\right)^{\mathrm{m}-1} \prod_{j=1}^{\mathrm{m}-2} \mathrm{X}_{3 j+1}^{(m-j-1)} \mathrm{T}_{3 j+4}^{(m-j-1)}, \mathrm{m} \geqslant 3, \\
& \frac{\chi_{1}\left(\left[\operatorname{sm}(x, \alpha) \mathrm{cm}^{2}(x, \alpha)\right]_{3 v+1}\right)}{\mathrm{H}_{1}^{(1)}\left(\left[\operatorname{sm}(x, \alpha) \mathrm{cm}^{2}(x, \alpha)\right]_{3 v+1}\right)}=-W_{1}^{*}, \\
& \frac{\chi_{m}\left(\left[\operatorname{sm}(x, \alpha) \mathrm{cm}^{2}(x, \alpha)\right]_{3 v+1}\right)}{H_{m}^{(1)}\left(\left[\operatorname{sm}(x, \alpha) \mathrm{cm}^{2}(x, \alpha)\right]_{3 v+1}\right)}=-\left[W_{1}^{*}+\sum_{j=1}^{m-1} W_{3 j+1}\right], \quad m \geqslant 2 .
\end{aligned}
$$

(iii)

$$
\begin{aligned}
& H_{1}^{(1)}\left(\left[\mathrm{sm}^{2}(x, \alpha) \mathrm{cm}^{2}(x, \alpha)\right]_{3 v+2}\right)=T_{2}^{*}, \\
& H_{m}^{(1)}\left(\left[\operatorname{sm}^{2}(x, \alpha) \mathrm{cm}^{2}(x, \alpha)\right]_{3 v+2}\right)=\left(T_{2}^{*}\right)^{m} \prod_{j=1}^{m-1} x_{3 j-1}^{(m-j)} T_{3 j+2}^{(m-j)}, \quad m \geqslant 2, \\
& \frac{\chi_{m}\left(\left[\operatorname{sm}^{2}(x, \alpha) \mathrm{cm}^{2}(x, \alpha)\right]_{3 v+2}\right)}{H_{m}^{(1)}\left(\left[\operatorname{sm}^{2}(x, \alpha) \mathrm{cm}^{2}(x, \alpha)\right]_{3 v+2}\right)}=-\sum_{j=1}^{m} W_{3 j-1}, \quad m \geqslant 1 .
\end{aligned}
$$

(iv) Let $\mathrm{N}=3,6,9,12, \cdots$

$$
\begin{aligned}
& H_{1}^{(1)}\left(\left[\mathrm{sm}^{\mathrm{N}}(x, \alpha) \mathrm{cm}^{2}(x, \alpha)\right]_{3 v+N}\right)=T_{3 i}, \\
& H_{m}^{(1)}\left(\left[\mathrm{sm}^{\mathrm{N}}(x, \alpha) \mathrm{cm}^{2}(x, \alpha)\right]_{3 v+N}\right)=\left(T_{3 i}\right)^{m} \prod_{j=1}^{m-1} x_{3 j+3 i-3}^{(m-j)} T_{3 j+3 i}^{(m-j)}, \quad m \geqslant 2, \\
& \frac{\chi_{m}\left(\left[\operatorname{sm}^{N}(x, \alpha) \mathrm{cm}^{2}(x, \alpha)\right]_{3 v+N}\right)}{H_{m}^{(1)}\left(\left[\operatorname{sm}^{N}(x, \alpha) \mathrm{cm}^{2}(x, \alpha)\right]_{3 v+N}\right)}=-\sum_{j=1}^{m} W_{3 j+3 i-3,}, \quad m \geqslant 1,
\end{aligned}
$$

where $i=1,2,3, \cdots, \frac{N}{3}$.

(v) Let $\mathrm{N}=4,7,10,13, \cdots$

$$
\begin{aligned}
& H_{1}^{(1)}\left(\left[\operatorname{sm}^{N}(x, \alpha) \mathrm{cm}^{2}(x, \alpha)\right]_{3 v+N}\right)=T_{3 i+1}, \\
& H_{m}^{(1)}\left(\left[\operatorname{sm}^{N}(x, \alpha) \mathrm{cm}^{2}(x, \alpha)\right]_{3 v+N}\right)=\left(T_{3 i+1}\right) \prod_{j=1}^{m-1} x_{3 j+3 i-2}^{(m-j)} T_{3 j+3 i+1}^{(m-j)}, \quad m \geqslant 2, \\
& \frac{x_{m}\left(\left[\operatorname{sm}^{N}(x, \alpha) \mathrm{cm}^{2}(x, \alpha)\right]_{3 v+N}\right)}{H_{m}^{(1)}\left(\left[\operatorname{sm}^{N}(x, \alpha) \mathrm{cm}^{2}(x, \alpha)\right]_{3 v+N}\right)}=-\sum_{j=1}^{m} W_{3 j+3 i-2,}, \quad m \geqslant 1,
\end{aligned}
$$

where $i=1,2,3, \cdots, \frac{N-1}{3}$.

(vi) Let $\mathrm{N}=5,8,11,14, \cdots$

$$
\begin{aligned}
& H_{1}^{(1)}\left(\left[\operatorname{sm}^{N}(x, \alpha) \mathrm{cm}^{2}(x, \alpha)\right]_{3 v+N}\right)=T_{3 i+2} \\
& H_{m}^{(1)}\left(\left[\operatorname{sm}^{N}(x, \alpha) \mathrm{cm}^{2}(x, \alpha)\right]_{3 v+N}\right)=\left(T_{3 i+2}\right) \prod_{j=1}^{m-1} x_{3 j+3 i-1}^{(m-j)} T_{3 j+3 i+2}^{(m-j)}, \quad m \geqslant 2,
\end{aligned}
$$




$$
\frac{\chi_{m}\left(\left[\operatorname{sm}^{N}(x, \alpha) \mathrm{cm}^{2}(x, \alpha)\right]_{3 v+N}\right)}{H_{m}^{(1)}\left(\left[\operatorname{sm}^{N}(x, \alpha) \mathrm{cm}^{2}(x, \alpha)\right]_{3 v+N}\right)}=-\sum_{j=1}^{m} W_{3 j+3 i-1}, \quad m \geqslant 1,
$$

where $i=1,2,3, \cdots, \frac{\mathrm{N}-2}{3}$.

Here $\mathrm{T}_{(.)}, \mathrm{W}_{(.)}, \mathrm{X}_{(.)}, \mathrm{T}_{0}^{*}, \mathrm{~W}_{0}^{*}, \mathrm{X}_{0}^{*}, \mathrm{~T}_{1}^{*}, \mathrm{~W}_{1}^{*}, \mathrm{X}_{1}^{*}$ and $\mathrm{T}_{2}^{*}$ are given by respective equations (3.43), (3.45), (3.46), (3.50), (3.52), (3.53), (3.54), (3.56), (3.57) and (3.58).

Proof. Maclaurin series of $\mathrm{sm}^{\mathrm{N}}(x, \alpha) \mathrm{cm}^{2}(x, \alpha) ; \mathrm{N} \geqslant 0$ is given by,

$$
\mathrm{sm}^{\mathrm{N}}(x, \alpha) \mathrm{cm}^{2}(x, \alpha)=\sum_{v=0}^{\infty} \frac{\left[\mathrm{sm}^{\mathrm{N}}(x, 0) \mathrm{cm}(x, 0)\right]_{3 v+\mathrm{N}} x^{3 v+N}}{(3 v+\mathrm{N}) !}, \quad \mathrm{N} \geqslant 0,
$$

where $\left[\mathrm{sm}^{\mathrm{N}}(x, \alpha) \mathrm{cm}^{2}(x, \alpha)\right]_{3 v+N} ; \mathrm{N} \geqslant 0$ denotes Maclaurin series coefficients in polynomials of $\alpha$. Now assume $\mathrm{V}_{0}^{*} \equiv 1, \mathrm{~V}_{1}^{*} \equiv 1$ and $\mathrm{V}_{\mathrm{N}} ; \mathrm{N} \geqslant 2 \equiv 1$ in Theorem 3.5 so that it satisfies Lemma 2.5. Applying coefficients of Theorem 3.5 in (2.6) of Lemma 2.5 gives the Hankel determinants for equations (3.36), (3.37), (3.38), (3.39), (3.40), (3.41) by respective enumerates of Theorem 4.5.

Remark 4.6. Theorem 4.5 appears for first time in this work.

\section{Conclusion}

QCAF expansions of $\operatorname{sm}^{\mathrm{N}}(x, \alpha) ; N \geqslant 4, \operatorname{sm}^{\mathrm{N}}(x, \alpha) \mathrm{cm}(x, \alpha) ; N \geqslant 3$ and $\operatorname{sm}^{\mathrm{N}}(x, \alpha) \mathrm{cm}^{2}(x, \alpha) ; N \geqslant 0$ were appears for the first time in this work to the literature reviewed. Hankel determinants results were checked with Maple and confirmed that the results were exact as well as coincides with the results of lower order associated continued fractions in literature. Remarks 4.2 and 4.4 ensures that the assumption of $c_{0}$ and $c_{n} ; n \geqslant 1$ in the denominator of QACF to 1 is correct in Hankel determinants calculations. Also the compact form of Hankel determinants results in this work will ease the implementation in any of the Computer Algebra Software (CAS). For the future study it remains the open query to find Sumudu transform of $\mathrm{cm}^{3}(x, \alpha)$ and other higher powers which leads to the four term recurrences. Next Hankel determinants calculations of QACF by restricting the assumptions made in this paper remains open query for further study.

\section{References}

[1] O. S. Adams, Elliptic functions applied to conformal world maps, US Government Printing Office, Washington, (1925). 1

[2] W. A. Al-Salam, L. Carlitz, Some determinants of Bernoulli, Euler and related numbers, Portugal. Math., 18 (1959), 91-99. 1

[3] R. Bacher, P. Flajolet, Pseudo-factorials, elliptic functions, and continued fractions, Ramanujan J., 21 (2009), 71-97. 1

[4] F. B. M. Belgacem, E. H. Al-Shemas, R. Silambarasan, Sumudu computation of the transient magnetic field in a lossy medium, Appl. Math. Inf. Sci., 11 (2017), 209-217. 1

[5] F. B. M. Belgacem, R. Silambarasan, A distinctive Sumudu treatment of trigonometric functions, J. Comput. Appl. Math., 312 (2017), 74-81. 1

[6] F. B. M. Belgacem, R. Silambarasan, Further distinctive investigations of the Sumudu transform, AIP Conf. Proc., 1798 (2017), 020025-1-020025-9. 1

[7] F. B. M. Belgacem, R. Silambarasan, Sumudu transform of Dumont bimodular Jacobi elliptic functions for arbitrary powers, AIP Conf. Proc., 1798 (2017), 020026-1-020026-9. 1

[8] L. Carlitz, Some orthogonal polynomials related to elliptic functions, Duke Math. J., 27 (1960), 443-459. 1

[9] E. V. F. Conrad, Some continued fraction expansions of Laplace transforms of elliptic functions, Thesis (Ph.D.)-The Ohio State University, ProQuest LLC, Ann Arbor, MI, (2002), 87 pages. 1, 2, 2, 2, 2.3, 2.4, 2, 3, 3.1, 3.2, 3.3, 3.4, 4, 4.2, 4.4

[10] E. V. F. Conrad, P. Flajolet, The Fermat cubic, elliptic functions, continued fractions, and a combinatorial excursion, Sém. Lothar. Combin., 54 (2005/07), 44 pages. 1

[11] A. C. Dixon, On the doubly periodic functions arising out of the curve $x^{3}+y^{3}-3 \alpha x y=1$, Quart. J. Pure Appl. Math., 24 (1890), 167-233. 1, 2, 2 
[12] A. C. Dixon, The elementary properties of the elliptic functions, with examples, Macmillan, London and New York, (1894). 1

[13] D. Dumont, Le parametrage de la courbe d'equation $x^{3}+y^{3}=1$, Une introduction elementaire aux fonctions elliptiques, preprint, (1988). 1

[14] H. Eltayeb, A. Kiliçman, On double sumudu transform and double Laplace transform, Malays. J. Math. Sci., 4 (2010), 17-30. 1

[15] H. Eltayeb, A. Kılıçman, R. P. Agarwal, On integral transforms and matrix functions, Abstr. Appl. Anal., 2011 (2011), 15 pages. 1

[16] M. E. H. Ismail, D. R. Masson, Some continued fractions related to elliptic functions, Continued fractions: from analytic number theory to constructive approximation, Columbia, MO, (1998), Contemp. Math., Amer. Math. Soc., Providence, RI, 236 (1999), 149-166. 1

[17] W. B. Jones, W. J. Thron, Continued fractions, Analytic theory and applications, With a foreword by Felix E. Browder, With an introduction by Peter Henrici, Encyclopedia of Mathematics and its Applications, AddisonWesley Publishing Co., Reading, Mass., (1980). 2, 2.1, 2.3, 2.4, 2

[18] A. Kiliçman, H. Eltayeb, On a new integral transform and differential equations, Math. Probl. Eng., 2010 (2010), 13 pages. 1

[19] A. Kllıçman, H. Eltayeb, Some remarks on the Sumudu and Laplace transforms and applications to differential equations, ISRN Appl. Math., 2012 (2012), 13 pages.

[20] A. Kiliçman, H. Eltayeb, K. A. M. Atan, A note on the comparison between Laplace and Sumudu transforms, Bull. Iranian Math. Soc., 37 (2011), 131-141.

[21] A. Kılıçman, V. G. Gupta, B. Shrama, On the solution of fractional Maxwell equations by Sumudu transform, J. Math. Res., 2 (2010), 147-151. 1

[22] J. C. Langer, D. A. Singer, The trefoil, Milan J. Math., 82 (2014), 161-182. 1

[23] D. F. Lawden, Elliptic functions and applications, Applied Mathematical Sciences, Springer-Verlag, New York, (1989). 1

[24] L. Lorentzen, H. Waadeland, Continued fractions with applications, Studies in Computational Mathematics, NorthHolland Publishing Co., Amsterdam, (1992). 2

[25] A. M. Mahdy, A. S. Mohamed, A. A. Mtawa, Implementation of the homotopy perturbation Sumudu transform method for solving Klein-Gordon equation, Appl. Math., 6 (2015), 617-628. 1

[26] S. C. Milne, Infinite families of exact sums of squares formulas, Jacobi elliptic functions, continued fractions, and Schur functions, Reprinted from Ramanujan J., 6 (2002), 7-149, With a preface by George E. Andrews, Developments in Mathematics, Kluwer Academic Publishers, Boston, MA, (2002). 1, 2.3, 2.4, 2

[27] T. Muir, A treatise on the theory of determinants, Revised and enlarged by William H. Metzler, Dover Publications, Inc., New York, (1960). 2.4

[28] M. A. Ramadan, M. S. Al-Luhaibi, Application of sumudu decomposition method for solving linear and nonlinear KleinGordon equations, Int. J. Soft Comput. Eng., 3 (2014), 138-141. 1

[29] J. Singh, D. Kumar, A. Kilicman, Application of homotopy perturbation Sumudu transform method for solving heat and wave-like equations, Malays. J. Math. Sci., 7 (2013), 79-95. 1

[30] H. S. Wall, Note on the expansion of a power series into a continued fraction, Bull. Amer. Math. Soc., 51 (1945), 97-105. 2.1

[31] H. S. Wall, Analytic theory of continued fractions, D. Van Nostrand Company, Inc., New York, N. Y., (1948). 2.1

[32] X.-J. Yang, A new integral transform with an application in heat-transfer problem, Therm. Sci., 20 (2016), S677-S681. 1

[33] X.-J. Yang, A new integral transform operator for solving the heat-diffusion problem, Appl. Math. Lett., 64 (2017), 193197. 1 